\title{
The added value of an assessment of the patient's hand grip strength to the comprehensive geriatric assessment in G8-abnormal older patients with cancer in routine practice
}

\author{
Michelle Lycke ${ }^{\mathrm{a}, \mathrm{b}}$, Lore Ketelaars ${ }^{\mathrm{c}}$, Evi Martens ${ }^{\mathrm{c}}$, Tessa Lefebvre ${ }^{\mathrm{a}, \mathrm{b}}$, Hans Pottel ${ }^{\mathrm{d}}$, Koen Van Eygen ${ }^{\mathrm{e}}$, \\ Lieselot Cool $^{\mathrm{a}}$, Lies Pottel $^{\mathrm{a}}$, Cindy Kenis ${ }^{\mathrm{f}}$, Patricia Schofield ${ }^{\mathrm{g}}$, Tom Boterberg ${ }^{\mathrm{b}}$, Philip R. Debruyne ${ }^{\mathrm{a}, \mathrm{g}, *}$ \\ a Department of Medical Oncology, Cancer Centre, General Hospital Groeninge, Kortrijk, Belgium \\ b Department of Radiotherapy and Experimental Cancer Research, Ghent University, Ghent, Belgium \\ c Department of Psychology, General Hospital Groeninge, Kortrijk, Belgium \\ d Department of Public Health and Primary Care @ Kulak, Catholic University Leuven Kulak, Kortrijk, Belgium \\ e Department of Haematology, Cancer Centre, General Hospital Groeninge, Kortrijk, Belgium \\ ${ }^{\mathrm{f}}$ Department of General Medical Oncology and Geriatric Medicine, University Hospitals Leuven, Leuven, Belgium \\ ${ }^{\mathrm{g}}$ Positive Ageing Research Institute, Faculty of Health, Social Care and Education, Anglia Ruskin University, Chelmsford, UK
}

\section{A R T I C L E I N F O}

\section{Article history:}

Received 30 March 2018

Received in revised form 13 September 2018

Available online $\mathrm{xxxx}$

\section{Keywords:}

Comprehensive geriatric assessment

Functional status

Hand grip strength

Survival

\begin{abstract}
A B S T R A C T
Objectives: A comprehensive geriatric assessment (CGA) is the key treatment approach to guide decisions in older patients with cancer. In this paper, the added value of an assessment of the patient's hand grip strength to predict survival in patients with an abnormal G8-questionnaire (G8) score is investigated.

Materials and Methods: Patients were screened by the G8, followed by a CGA in case of an abnormal screening $(\leq 14.0)$. Hand grip strength was assessed by use of the JAMAR® hydraulic hand dynamometer. Cut-offs were applied according to the Fried frailty criteria. The survival rate was calculated twelve months after the CGA date. Results: We retrospectively reviewed data of 2071 patients who were treated at the Kortrijk Geriatric Oncology Clinic (General Hospital Groeninge, Belgium) between November 2012 and December 2016. Of those, 944 patients with a mean age of 79.6 years were included in the analyses. $64.2 \%$ of patients presented an abnormal hand grip strength score. A log rank test revealed a statistical significant result between patients when accounting for the hand grip strength score $(p<.01)$. When added to a Cox regression model, a significant result was found $(p<.01)$. However, this added only $0.4 \%$ to the explained variance of the model.

Discussion: While a statistically significant result was detected, when adding the hand grip strength score to a regression model for survival, our data indicate that such assessment may clinically be less relevant when included in an already extensive test battery and may therefore provide only limited information in terms of patient survival.
\end{abstract}

(c) 2019 Published by Elsevier Ltd.

\section{Introduction}

In older patients with cancer, identifying predictors for treatment toxicity as well as an increased morbidity and mortality is essential in order to increase the patient's overall survival and quality of life. For this reason, geriatric oncology guidelines have emphasized the need for individual treatment approaches in this heterogeneous population $[1,2]$. Treatment decisions should be guided by an in-depth evaluation of the patient's health by use of a comprehensive geriatric assessment (CGA). A CGA can be defined as a multidisciplinary evaluation in which multiple health domains are covered and aims at providing a

* Corresponding author at: General Hospital Groeninge, Cancer Centre, President Kennedylaan 4, B-8500 Kortrijk, Belgium.

E-mail address: Philip.Debruyne@azgroeninge.be (P.R. Debruyne). concise image of the patient's strengths and resources [3-7]. It further aims at developing a coordinated care plan to focus interventions and long-term follow-up on the person's detected vulnerabilities [8].

One of the domains covered within the CGA is the patient's functional capabilities. This assessment often includes an evaluation of the patient's daily activities, followed by an evaluation of the patient's history of falls and gait. The latter is often assessed by use of the Timed Up and Go test (TUG). In the TUG test, the patient is observed and timed while he rises from an arm chair, walks three meters, turns, walks back, and sits down again [9]. Though adequately for assessing the patient's physical reserve, other methods could be used to assess the patient's physical reserve. Upper limbs play an important role in everyone's daily life. A number of sensorimotor parameters, such as grip strength, are necessary for their optimal performance. Grip strength is therefore an important prerequisite for good functional 
performance [10]. Several studies have shown an association between hand grip strength and mortality and morbidity [11]. In a group of patients without cancer, poor grip strength is reported as an indicator of increased postoperative complications, prolonged hospitalization and decreased functional status. In older patients in particular, loss of grip strength may result in a loss of independence [12]. Further, poor grip strength is listed as one of the criteria to define frailty in older persons according to the National Comprehensive Cancer Network (NCCN) Guidelines in Older Adult Oncology [1]. A recent trial by Velghe et al. in older patients with haematological cancer reported that hand grip strength may be used as a screening tool to detect patients with an abnormal CGA [13]. Further, a trial by Versteeg et al. reported that higher muscle strength is associated with longer overall survival in older patients with advanced cancer [14].

As these researchers have reported on the benefits of an assessment of the patient's hand grip strength; in this paper, we aimed to define its added value as part of the CGA in older patients with cancer.

\section{Materials and Methods}

\subsection{Patients}

Data was collected as part of a routine assessment in older patients with cancer at the General Hospital Groeninge, Kortrijk, Belgium. Data was partly registered as part of a large multicentre registration trial aiming at the implementation of a nationwide uniform CGA or as part of routine registration in registered CGA databases. For both registration trials (AZGS2012057 and AZGS2015081), approval of the ethical committee was obtained.

Patients were recruited upon presentation at the Kortrijk Geriatric Oncology Clinic (General Hospital Groeninge). Eligible patients were 70 years or older at the time of inclusion and needed to be newly diagnosed with a solid tumour or haematological malignancy, or needed to be scheduled for a new line of treatment. Any type of stage and any type of treatment was allowed.

\subsection{Measures}

Patients were screened by the G8-questionnaire (G8) [15], followed by a CGA in case of an abnormal G8 result $(\leq 14.0)$. The CGA comprised following standardized measures: nutrition (Mini Nutritional Assessment - Short Form (MNA-SF) [16]), functional status (Activities of Daily Living (ADL), Instrumental Activities of Daily Living (iADL), history of falls [17,18]), emotional status (Geriatric Depression scale - 15 (GDS) [19]), cognition (Mini Mental State Examination (MMSE) or Freund Clock Drawing Test (CDT) [20,21]), polypharmacy (number of drugs), and comorbidities (Charlson Comorbidity Index (CCI) [22]).

Functional status was further assessed through the patient's hand grip strength [23]. To assess hand grip strength, the JAMAR® hydraulic hand dynamometer was used. For this test, patients were asked to sit with their shoulder adducted and neutrally rotated, elbow flexed at $90^{\circ}$, forearm in neutral position, and wrist between $0^{\circ}$ and $30^{\circ}$ dorsiflexion and between $0^{\circ}$ and $15^{\circ}$ ulnar deviation. After proper positioning, the patients were asked to squeeze the dynamometer as hard as possible with their dominant hand. The final JAMAR® or hand grip strength score, presented in kilograms ( $\mathrm{kg})$, was calculated as the average score of three successive attempts [24]. Hand grip strength vulnerability was defined by the fried frailty criteria and were dependent of the patient's gender and body mass index as shown in Table 1 .

The survival rate was obtained through identification in the patients' medical records twelve months after the CGA date.

\subsection{Statistical Considerations}

Descriptive statistics were performed to present patient and tumour characteristics and CGA results. The definition of CGA vulnerability was
Table 1

Hand grip strength according to Fried et al. [24].

\begin{tabular}{lllll}
\hline Male & & & Female \\
\cline { 5 - 5 } BMI & Cut-off $(\mathrm{kg})$ & & BMI & Cut-off $(\mathrm{kg})$ \\
\hline$\leq 24$ & $\leq 29$ & $\leq 23$ & $\leq 17$ \\
$24.1-26$ & $\leq 30$ & $23.1-26$ & $\leq 17.3$ \\
$26.1-28$ & $\leq 30$ & $26.1-29$ & $\leq 18$ \\
$>28$ & $\leq 32$ & $>29$ & $\leq 21$ \\
\hline
\end{tabular}

Abbreviations: BMI: Body Mass Index

based on previous research and defined as meeting the cut-off scores for impairment in two or more CGA domains as this an impairment in $\geq 2$ domains leads for example to an increased risk for future disability or mortality [25]. Functional impairment was defined as an abnormal test score on either the ADL or iADL. Patients were marked as having a cognitive impairment with following approach: vulnerability was defined as having an abnormal test score on either the CDT or MMSE; in case both tests were assessed, the score of the MMSE overruled the score of the CDT. The hand grip strength score was not included to define CGA vulnerability as its added value has not yet been established. Hand grip strength vulnerability was defined by the Fried frailty criteria as reported in previous NCCN Guidelines [26].

An independent student-t-test was used to compare hand grip strength scores between men and women as well as between alive and deceased patients.

The added value of the hand grip strength score further was analysed by use of both a Cox regression analysis as well as binary logistic regression analyses. First, a Cox regression analysis, corrected for age and gender was conducted, looking into the predictive value of CGA variables for survival twelve months after the CGA assessment. The analysis included following independent variables: type of diagnosis (new diagnosis, relapse or progression), treatment intent, ADL score, iADL score, GDS score, falls during the past year (yes or no), MNA-SF score, CCI score, number of drugs, and cognitive impairment. A final model was selected through forward and backward analyses as well as analyses of individual variables. Variables were allowed in the model at the $p<.05$ level. Once the best model was selected, the hand grip strength score (normal or abnormal) was added to the analysis. We stated to acknowledge the added value of the hand grip strength if it remained present in the final model. The added value of the hand grip strength score was further evaluated by use of a binary logistic regression analysis and defined in terms of the increase in the Nagelkerke $\mathrm{R}^{2}$ value, reporting the explained variance of the selected model.

\section{Results}

We retrospectively reviewed data of 2071 patients who visited the Kortrijk Geriatric Oncology Clinic between November 2012 and December 2016. Of those patients, 861 patients were excluded from the analysis as they presented with a normal G8 score $(\geq 14.5)$. Another 260 patients were excluded due to missing data. Last, six patients had to be excluded as they were not diagnosed with a malignant condition after all (Fig. 1).

Eligible patients had a mean age of 79.6 years (range: 70-99) and included slightly more male patients (52.1\%). Most patients were married (55.7\%) and lived at home with their partner (53.2\%) (Table 2). The majority of patients received a new diagnosis $(82.2 \%)$ of a solid tumour or haematological malignancy, whereas respectively $12.0 \%$ and $5.8 \%$ of patients presented with a disease progression or relapse. Eligible patients were diagnosed with cancer of following sites: digestive (33.5\%), genitourinary $(16.7 \%)$, breast $(12.4 \%)$, haematological malignancy $(11.0 \%)$, thorax (10.0\%), gynaecological (8.7\%), head and neck (3.9\%), skin (1.7\%), central nervous system $(0.8 \%)$, musculoskeletal $(0.7 \%)$, or unknown primary $(0.6 \%)$ (Table 3$)$. Twelve-month survival was $64.1 \%$. 


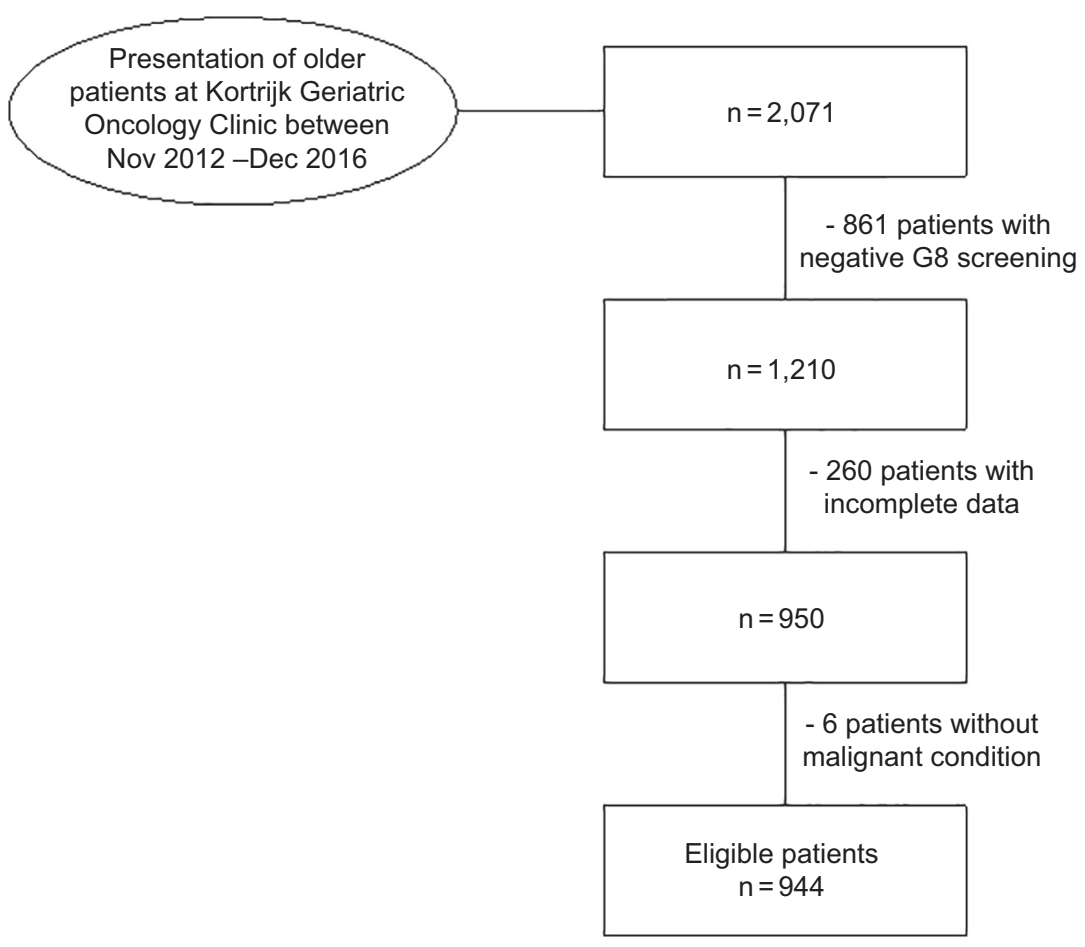

Fig. 1. Flowchart of included patients in analyses.

Table 4 represents mean scores and percentage vulnerability of each domain included in the CGA. Most vulnerabilities were detected in the domains of functional status (89.3\%), nutrition (73.0\%), and polypharmacy (69.8\%). Almost all patients (92.0\%) were deemed vulnerable according to the CGA. According to the Fried frailty criteria, $64.2 \%$ of patients presented a poor hand grip strength score. Male individuals had a significantly higher mean hand grip strength compared to women ( $p<.001 ; 28.1 \mathrm{~kg}$ and $16.6 \mathrm{~kg}$, respectively). When comparing hand grip strength scores between patients who were alive and who passed away after twelve months, significantly higher hand grip strength scores were noted in the twelve-month survivors in both men and women (men: $29.5 \mathrm{~kg}$ vs. $26.0 \mathrm{~kg}, p<.001$; women: $17.1 \mathrm{~kg}$ vs. $15.3 \mathrm{~kg}, p<.01$ ). A chi square test detected a significant difference when comparing normal and abnormal JAMAR ${ }^{\circledR}$ tests results between alive and deceased patients for the whole patient group $(p<.001)$.

Table 2

Patient characteristics.

\begin{tabular}{lll}
\hline Characteristics $(n=944)$ & Mean (range) & $\mathrm{n}(\%)$ \\
\hline Age (years) & $79.6(70-99)$ & \\
Sex & & $452(47.9)$ \\
Female & $492(52.1)$ \\
$\quad$ Male & \\
Marital Status & $526(55.7)$ \\
Married & $334(35.4)$ \\
Widow-er & $43(4.6)$ \\
Single & $22(2.3)$ \\
Divorced & $12(1.3)$ \\
Other & $7(0.7)$ \\
Legally cohabiting & \\
Living situation & $502(53.2)$ \\
Home with partner & $311(32.9)$ \\
Home - alone & $40(4.2)$ \\
Institution & $39(4.1)$ \\
Home with family member & $37(4.0)$ \\
Service flat & $15(1.6)$ \\
Other &
\end{tabular}

Fig. 2 represents the Kaplan Meier curve compared for patients with normal and abnormal hand grip strength score showing higher survival rates after one year in patients with a normal hand grip strength score. The log rank test revealed a statistical significant difference between both groups $(p<.01)$. The Cox regression analysis looking into predictive CGA variables for twelve-month survival, revealed a final model, corrected for age and gender, with following variables: treatment intent and MNA-SF score (Table 5). The explained variance of this model by use of the Nagelkerke $\mathrm{R}^{2}$ was $24.0 \%$ by use of a binary regression analysis. When adding the hand grip strength score to this model, an added explained variance of $0.4 \%$ was found, leading to a total Nagelkerke $\mathrm{R}^{2}$ of $24.4 \%$ (Table 5 ).

Table 3

Tumour characteristics.

\begin{tabular}{ll}
\hline Characteristics $(\mathrm{n}=944)$ & $\mathrm{n}(\%)$ \\
\hline Cancer site & \\
Digestive & $315(33.5)$ \\
Genitourinary & $158(16.7)$ \\
Breast & $117(12.4)$ \\
Hematologic malignancy & $104(11.0)$ \\
Thorax & $94(10.0)$ \\
Gynaecological & $82(8.7)$ \\
Head and neck & $37(3.9)$ \\
Skin & $16(1.7)$ \\
Central nervous system & $8(0.8)$ \\
Musculoskeletal & $7(0.7)$ \\
Unknown primary & $6(0.6)$ \\
Diagnosis & \\
New diagnosis & $776(82.2)$ \\
Progression & $113(12.0)$ \\
Relapse & $55(5.8)$ \\
Treatment intent & \\
Curative & $542(57.4)$ \\
Palliative & $320(33.9)$ \\
No active treatment & $82(8.7)$
\end{tabular}


Table 4

Overview of median scores and percentage of vulnerable patients per tool.

\begin{tabular}{lllll}
\hline Domain & Test & Range & Mean & $\begin{array}{l}\% \\
\text { vulnerable }\end{array}$ \\
\hline Nutrition & MNA-SF $(\mathrm{n}=944)$ & $0-14$ & 9.7 & 73.0 \\
Functional status & ADL $(n=943)$ & $0-24$ & 8.8 & 89.3 \\
& IADL $(\mathrm{n}=943)$ & $0-8$ & 4.6 & \\
& History of falls & NA & NA & \\
Depression & GDS $(n=943)$ & $0-15$ & 2.3 & 16.8 \\
Cognition & MMSE $(n=539)$ & $0-30$ & 24.8 & 25.3 \\
& CDT $(n=388)$ & $0-7$ & 4.7 & 69.8 \\
Polypharmacy & Number of drugs & NA & 6.6 & 13.3 \\
Comorbidities & CCI $(\mathrm{n}=943)$ & & &
\end{tabular}

Abbreviations: MNA-SF: Mini Nutritional Assessment-Short Form, ADL: Activities of Daily Living, IADL: Instrumental Activities of Daily Living, GDS: Geriatric Depression Scale, MMSE: Mini Mental State Examination, CDT: Clock Drawing Test, CCI: Charlson Comorbidity Index, NA: Not applicable.

\section{Discussion}

A CGA is the cornerstone in modern geriatric oncology. In this paper, we evaluated the added value of an assessment of the patient's hand grip strength by looking at its predictive value for twelve-month survival.

Our results indicate that an assessment of the hand grip strength by use of the JAMAR® hydraulic hand dynamometer can predict survival twelve months after the CGA assessment as a significant result was found when the hand grip strength score was added to an optimised Cox regression analysis. Adding the hand grip strength score to a CGA assessment will lead to a $0.4 \%$ increase in the explained variance of our selected model to predict survival. These results indicate that higher muscle strength is associated with longer survival in our target population and confirm findings of Versteeg et al. [14]). In line with the work of Velghe et al., our results also indicate that an assessment of the patient's hand grip strength can predict vulnerability on the CGA as patients with a normal CGA had a significantly lower hand grip strength score (data not shown) [13]. As a logical consequence, an abnormal hand grip strength score is also associated with lower G8 scores in normal screening patients (data not shown).

While these results indicate that an assessment of the patient's hand grip strength adds some value to the CGA, one could argue whether

\section{Table 5}

Overview of variables included in the final regression analyses predicting 12-month survival.

\begin{tabular}{|c|c|c|c|c|}
\hline \multirow[b]{2}{*}{ Cox regression } & \multicolumn{2}{|c|}{$\begin{array}{l}\text { Model without hand } \\
\text { grip strength score }\end{array}$} & \multicolumn{2}{|c|}{$\begin{array}{l}\text { Model with hand grip } \\
\text { strength score }\end{array}$} \\
\hline & $\beta$ & $p$-value & $\beta$ & $p$-value \\
\hline MNA-SF total score & -0.150 & 0.000 & -0.147 & 0.000 \\
\hline Treatment intent & 0.838 & 0.000 & 0.822 & 0.000 \\
\hline Hand grip strength score & - & - & 0.340 & 0.009 \\
\hline Logistic regression & \multirow{2}{*}{\multicolumn{2}{|c|}{0.240}} & & \\
\hline Nagelkerke $\mathrm{R}^{2}$ & & & \multicolumn{2}{|l|}{0.244} \\
\hline
\end{tabular}

Abbreviations: MNA-SF: Mini Nutritional Assessment-Short Form.

these positive statistical results are of high clinical significance. For instance, the hand grip strength score was not selected in the initial final regression model. As a trial by Norman et al. (2010) reports that malnutrition emerges as an independent determinant for hand grip strength, a post hoc correlation analysis was conducted, clearly indicating a positive correlation between both variables $(r=0.190, p<.001)$ [27]. Therefore, intercollinearity between the MNA-SF score and the hand grip strength score have led to a significant result when the latter was added to the regression model. Further, when looking at the explained variance of both variables independently, the hand grip strength score shows a Nagelkerke $\mathrm{R}^{2}$ of $3.0 \%$, while the MNA-SF has an explained variance of $10.6 \%$ (both models corrected for age and gender; data not shown). As the MNA-SF has a higher Nagelkerke $\mathrm{R}^{2}$ value, one could state that an assessment of the MNA-SF alone may be sufficient to predict twelve-month survival in our group of older patients with cancer.

However, a review by Norman et al. reported that hand grip strength has become a popular marker for the patient's nutritional status as it is quickly affected by nutritional deprivation [12]. Therefore, one could state that an assessment of the patient's hand grip strength should be added to the G8. As the G8 contains multiple questions from the MNA-SF, another post hoc regression analysis was conducted looking at the explained variance of the G8 alone. A binary logistic regression analysis for survival, corrected for age and gender, showed a Nagelkerke $\mathrm{R}^{2}$ value of $12.7 \%$. Since this value is even higher than the explained variance of the MNA-SF alone, these results indicate that the G8 has the highest capacity of predicting the patient's survival twelve months post CGA. This result is not surprising as other researchers have already

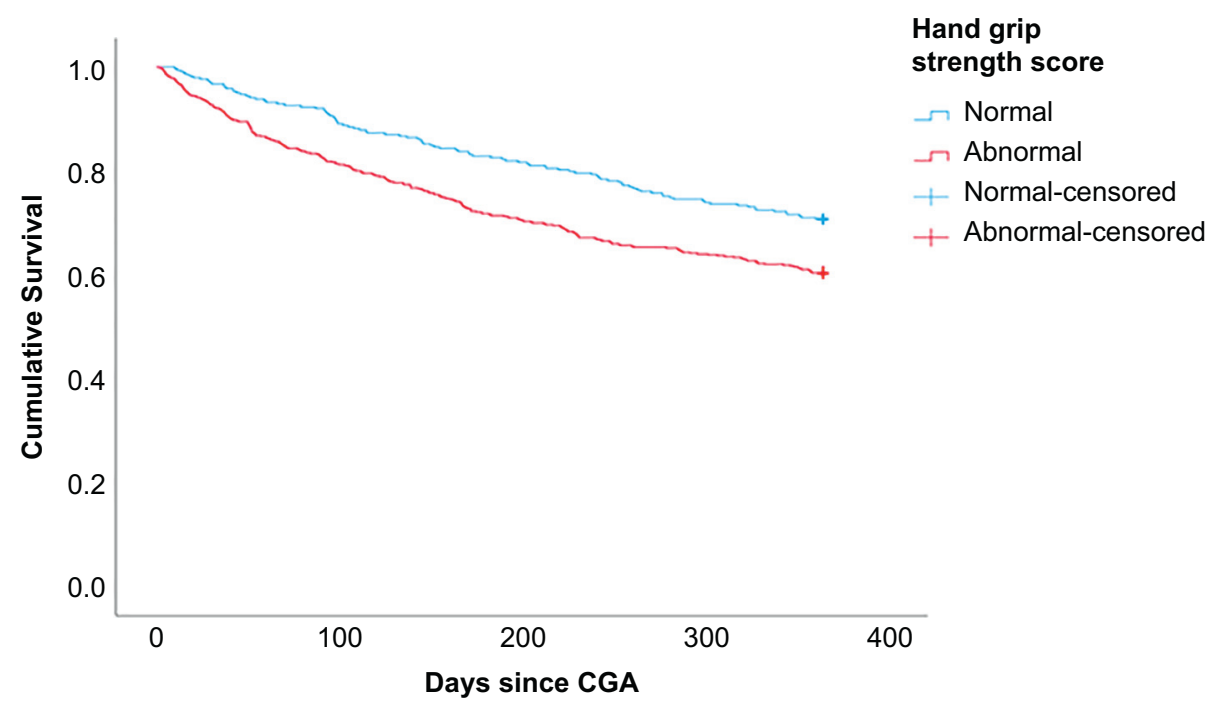

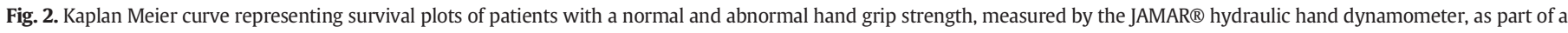
comprehensive geriatric assessment (CGA) in G8-abnormal patients. 
published data on the prognostic value of the G8 on overall survival $[15,28]$. Further, the G8 alone also has a higher explained variance for CGA vulnerability in our selected patient group compared the hand grip strength score alone ( $21.6 \%$ vs. $5.2 \%$ ).

These results, however, do not indicate that one should perform a G8 screening alone. The found Nagelkerke $R^{2}$ value is low, indicating that other variables influence the patient's survival. Further, it should be noted, that the questionnaires included in the CGA, have an important informative message and that an assessment of the different domains is necessary in order to establish a tailored treatment plan. Based on the CGA, health care workers and physicians can detect problems or vulnerabilities in the several health domains. Consecutively, when such vulnerability is detected, a proper referral should be made in order to decrease the risk on morbidity and mortality. While our data show that some questionnaires, assessed within the CGA, may only have a limited contribution in predicting the patient's survival, they may negatively affect survival if detected vulnerabilities are left untreated.

The results reported in this paper need to be interpreted with caution due to some limitations. All patients included in the analysis had to have an abnormal G8 score as a full CGA would otherwise not have been conducted. The results may therefore only have an implication for patients with an abnormal screening. Nonetheless, one would expect that patients with a normal G8 screening have a sufficient amount of hand grip strength. Further, our data are in line with previous research, indicating that the G8 is the best tool to predict survival in older patients with cancer [28]. Therefore, we may extrapolate findings regarding the G8 score to patients with a normal G8 score. Another limitation regards the study design. Since the results presented in this manuscript were retrospectively calculated, we had to exclude a large number of patients without a hand grip strength score or who were not fully assessed by the CGA. We further did not find a normal distribution of cancer types. Nevertheless, as we were able to include a large number of patients and as our findings are in line with those of other researchers, our results are representative for the whole geriatric oncology population in Belgium. Last, we should mention that in some cases, it is not possible to conduct an assessment of the patient's hand grip strength: for example in patients who have an intravenous infusion in their dominant hand, in breast cancer patients who received surgery on the ipsilateral side of their dominant hand, in patients who have an arthrosic or rheumatic condition that affects their hand mobility.

To the best of our knowledge, we are the first to report data on the added value of an assessment of the hand grip strength in G8abnormal, older patients with cancer as part of a CGA. Further, as we were able to include a large number of patients, we may state that these results concern all patients who have an abnormal G8 score and who were consecutively assessed by a CGA. Future research, however, could focus on the added value of an assessment of the patient's hand grip strength in both normal and abnormal screening patients and should include a comparison with other physical measures.

In conclusion, while the hand grip strength score shows statistically significant results for predicting CGA vulnerability, our data indicate that such assessment is clinically less relevant when included in an already extensive test battery and may therefore provide only limited information in terms of patient survival.

\section{Conflict of Interest}

The authors have no conflict of interest to declare.

\section{Contributions}

All authors have contributed substantially to the design, acquisition of data and/or the analyses of data. All authors have reviewed and approved this manuscript.

\section{Acknowledgements}

Our work was supported by the Cancer Plan Actions of the Belgian Federal Public Service of Health, Food Chain Safety and Environment (NKP_24_018, KPC_24_A_025, KP_CA_04 and KPC_2122C_044).

\section{References}

[1] NCCN Practice guidelines in older Adult Oncology Version 2, Vol. 2017; 2017.

[2] Lycke M, Pottel L, Boterberg T, Ketelaars L, Wildiers H, Schofield P, et al. Integration of geriatric oncology in daily multidisciplinary cancer care: the time is now. Eur J Cancer Care (Engl) 2015;24(2):143-6.

[3] Brunello A, Sandri R, Extermann M. Multidimensional geriatric evaluation for older cancer patients as a clinical and research tool. Cancer Treat Rev 2009;35(6):487-92.

[4] Solomon DH, Judd HL, Sier HC, Rubenstein LZ, Morley JE. New issues in geriatric care. Ann Intern Med 1988;108(5):718-32.

[5] Lycke M, Boterberg T, Martens E, Ketelaars L, Pottel H, Lambrecht A, et al. Implementation of uHear - an iOS-based application to screen for hearing loss - in older patients with cancer undergoing a comprehensive geriatric assessment. J Geriatr Oncol 2016;7(2):126-33.

[6] Lycke M, Debruyne PR, Lefebvre T, Martens E, Ketelaars L, Pottel H, et al. The use of uHear to screen for hearing loss in older patients with cancer as part of a comprehensive geriatric assessment. Acta Clin Belg 2017:1-7.

[7] Pottel L, Lycke M, Boterberg T, Pottel H, Goethals L, Duprez F, et al. Serial comprehensive geriatric assessment in elderly head and neck cancer patients undergoing curative radiotherapy identifies evolution of multidimensional health problems and is indicative of quality of life. Eur J Cancer Care (Engl) 2014;23(3):401-12.

[8] Rubenstein LZ, Stuck AE, Siu AL, Wieland D. Impacts of geriatric evaluation and management programs on defined outcomes: overview of the evidence. J Am Geriatr Soc 1991;39(9) [Pt 2):8S-16S; discussion 7S-8S].

[9] Pondal M, del Ser T. Normative data and determinants for the timed "up and go" test in a population-based sample of elderly individuals without gait disturbances. J Geriatr Phys Ther 2008;31(2):57-63.

[10] Taekema DG, Gussekloo J, Maier AB, Westendorp RGJ, de Craen AJM. Handgrip strength as a predictor of functional, psychological and social health. A prospective population-based study among the oldest old. Age Ageing 2010;39(3):331-7.

[11] De Buyser SL, Petrovic M, Taes YE, Toye KR, Kaufman JM, Goemaere S. Physical function measurements predict mortality in ambulatory older men. Eur J Clin Invest 2013;43(4):379-86.

[12] Norman K, Stobaus N, Gonzalez MC, Schulzke JD, Pirlich M. Hand grip strength: outcome predictor and marker of nutritional status. Clin Nutr 2011;30(2):135-42.

[13] Velghe A, De Buyser S, Noens L, Demuynck R, Petrovic M. Hand grip strength as a screening tool for frailty in older patients with haematological malignancies. Acta Clin Belg 2016;71(4):227-30

[14] Versteeg KS, Blauwhoff-Buskermolen S, Buffart LM, de van der Schueren MAE, JAE Langius, HMW Verheul, et al. Higher muscle strength is associated with prolonged survival in older patients with advanced cancer. Oncologist 2017 Epub 2017 Dec 8.

[15] Pottel L, Lycke M, Boterberg T, Pottel H, Goethals L, Duprez F, et al. G-8 indicates overall and quality-adjusted survival in older head and neck cancer patients treated with curative radiochemotherapy. BMC Cancer 2015;15:875.

[16] Rubenstein LZ, Harker JO, Salva A, Guigoz Y, Vellas B. Screening for undernutrition in geriatric practice: developing the short-form mini-nutritional assessment (MNASF). J Gerontol A Biol Sci Med Sci 2001;56(6):M366-72.

[17] Katz S, Ford AB, Moskowitz RW, Jackson BA, Jaffe MW. Studies of Illness in the Aged. The index of Adl: a standardized measure of biological and psychosocial function. JAMA 1963;185:914-9.

[18] Lawton MP, Brody EM. Assessment of older people: self-maintaining and instrumental activities of daily living. Gerontologist 1969;9(3):179-86.

[19] Yesavage JA. Geriatric Depression Scale. Psychopharmacol Bull 1988;24(4):709-11.

[20] Folstein MF, Folstein SE, McHugh PR. "Mini-mental state". A practical method for grading the cognitive state of patients for the clinician. J Psychiatr Res 1975;12(3): 189-98.

[21] Freund B, Gravenstein S, Ferris R, Burke BL, Shaheen E. Drawing clocks and driving cars. J Gen Intern Med 2005;20(3):240-4.

[22] Charlson M, Szatrowski TP, Peterson J, Gold J. Validation of a combined comorbidity index. J Clin Epidemiol 1994;47(11):1245-51.

[23] American Society of Hand Therapists. Clinical Assessment Recommendations Chicago ; 1992.

[24] Mathiowetz V, Rennells C, Donahoe L. Effect of elbow position on grip and key pinch strength. J Hand Surg Am 1985;10(5):694-7.

[25] Mohile SG, Bylow K, Dale W, Dignam J, Martin K, Petrylak DP, et al. A pilot study of the vulnerable elders survey-13 compared with the comprehensive geriatric assessment for identifying disability in older patients with prostate cancer who receive androgen ablation. Cancer 2007;109(4):802-10.

[26] Fried LP, Tangen CM, Walston J, Newman AB, Hirsch C, Gottdiener J, et al. Frailty in older adults: evidence for a phenotype. J Gerontol A Biol Sci Med Sci 2001;56(3): M146-56.

[27] Norman K, Stobaus N, Smoliner C, Zocher D, Scheufele R, Valentini L, et al. Determinants of hand grip strength, knee extension strength and functional status in cancer patients. Clin Nutr 2010;29(5):586-91.

[28] Kenis C, Decoster L, Van Puyvelde K, De Greve J, Conings G, Milisen K, et al. Performance of two geriatric screening tools in older patients with cancer. J Clin Oncol 2014;32(1):19-26 